\title{
Acetylcholinesterase Based Detection of Residual Pesticides on Cotton
}

\author{
Syed Zameer Ul Hassan, Jiri Militky \\ Department of Textile Materials, Technical University of Liberec, Liberec, Czech Republic \\ Email: zameer551214@hotmail.com
}

Received December 1, 2011; revised January 5, 2012; accepted January 12, 2012

\begin{abstract}
This study describes the measurement of bio-electrical signals caused by enzymatic inhibition of acetylcholinesterase $(\mathrm{AChE})$ for the detection of organophosphorous and carbamate pesticides which are the strong inhibitors of AChE and prevents its normal function of the rapid removal of acetylcholine (Ach). Biosensor Toxicity Analyzer (BTA) was used for the testing and enzyme activity was determined by acetylthiocholine chloride (ATCCl) as enzyme substrate. The monitoring of changes in bio-electrical signals caused by the interaction of biological substances and residues were evaluated. Two samples of cotton were analyzed. Cryogenic homogenization was carried out for sample pretreatment and Soxhlet extraction method (SOX) was used for extraction. The resulted extracts were concentrated and then injected in the BTA. The method shows reasonable results and can successfully be utilized for the detection of residual pesticides on different types of cotton.
\end{abstract}

Keywords: Acetylcholinesterase; Acetylcholine; Acetylthiocholine Chloride; Biosensor Toxicity Analyzer; Cryogenic Homogenization; Soxhlet Extraction Method

\section{Introduction}

Cotton has been part of the fabric of human existence for thousands of years. Cotton not only produces the natural fibers used in textiles and clothing but also yields a high grade vegetable oil [1]. Cotton today provides almost $38 \%$ of the world textile consumption, second only to polyester, which recently took the lead [2]. Cotton production is highly technical and difficult because of pest pressures and environment, e.g. drought, temperature and soil nutritional conditions. The total area dedicated to cotton production accounts approximately $2.4 \%$ of arable land globally and cotton accounts for an estimated $16 \%$ of the world's pesticide consumption [3]. Pesticides are widely used for the control of weeds, diseases, and pests all over the world, mainly since after Second World War, and at present, around 2.5 million tons of pesticides are used annually and the number of registered active substances is higher than 500. Humans can be exposed to pesticides by direct or indirect means. Direct or primary exposure normally occurs during the application of these compounds and indirect or secondary exposure can take place through the environment or the ingestion of food [4].

This is why development of natural biological methods of insect control was initiated. Cotton grown without the use of insect control was initiated. Cotton grown without the use of any synthetically compounded chemicals (i.e. pesticides, fertilizers, defoliants, etc.) is considered as "organic" cotton. It is produced under a system of production and processing that seeks to maintain soil fertility and the ecological environment of the crop [5]. Pesticides are toxic compounds that may cause adverse effects on the human and the environment. Benzoylureas, carbamates, organophosphorous compounds, pyrethroids, sulfonylureas and triazines are the most important groups [6]. The organophosphates and carbamates are powerful inhibitors of acetylcholinesterase [7]. They can irreversibly inhibit acetylcholinesterase (AChE) which is essential for the function of the central nervous system [8], resulting in the buildup of the neurotransmitter acetylcholine which interferes with muscular responses and in vital organs produce serious symptoms and eventually death [9].

Inhibition of $\mathrm{AChE}$ by any xenobiotic compound is used as a tool for assessment of toxicity of some pesticides such as organophosphates and carbamates [10]. As the pesticide residue is a potentially serious hazard to human health, the control and detection of pesticide residue plays a very important role in minimizing risk. Many methods have been developed in the last few years for the detection of organophosphorous pesticides. The most widely used methods are gas chromatography (GC), high-performance liquid chromatography (HPLC), gas chromatography-mass spectrometry (GC-MS), immune assay and fluorescence. However, these techniques, which are time consuming, expensive and require highly trained personnel, are avail- 
able only in sophisticated laboratories [11].

Biosensors based on the inhibition of acetylcholine esterase (AChE) have been widely used for the detection of OP compounds [12]. Electro analytical sensors and biosensors provide an exciting and achievable opportunity to perform biomedical, environmental, food and industrial analysis away from a centralized laboratory due to their advantages such as high selectivity and specificity, rapid response, low cost of fabrication, possibility of miniaturization and easy to integrate in automatic devices [13]. Electrochemical biosensors for measurement of these pesticides are based on the inhibition of $\mathrm{AChE}$ and the inhibition degree is proportional to the pesticide concentration [14].

In this paper, we report another method for the determination of Organophosphorous and Carbamates pesticides based on acetylcholinesterase inhibition using AC1.W2.R1/ACCHE sensors with the help of Biosensor Toxicity Analyzer (BTA). Compared with other kinds of electrochemical AChE biosensors, this method is simple, fast and more sensitive for pesticide determination with much lower detection limit.

\section{Principle of BTA}

The target for many insecticides is an enzyme called acetylcholinesterase (AChE) [15]. Acetylcholinesterase's (AChE) biological role is the termination of impulse transmissions at cholinergic synapses within the nervous system of the insects and mammals by rapid hydrolysis of the neurotransmitter acetylcholine. Pesticides block the catalytic activity of the active center, thus acting as inhibitors of AChE. This results in the accumulation of acetylcholine in the synaptic membrane, which blocks the nerves to process the signals properly [16].

Biosensor toxicity analyzer (BTA) works on the above mentioned principle and monitors the activity of the inhibition of AChE with the help of sensors which are equipped with an enzymatic membrane of AChE enzyme which is immobilized. It consists of two major parts, one of which is the Microflow unit and the other is Bioanalyzer. The microflow unit has the capillary arrangement which allows precise and constant flow of the liquid onto the active surface of the AChE sensor for a high level of repeatability and sensitivity in the measurements. The module has an integrated chamber in which the sensor can easily be placed or replaced as shown in Figure 1.

\section{Experimental}

\subsection{Materials}

Two samples of Egyptian cotton variety Giza 86 were collected from the cultivation season 2009/2010. One of them was the classical conventional cotton and the other was ornic cotton without utilizing the synthetic pesticides.

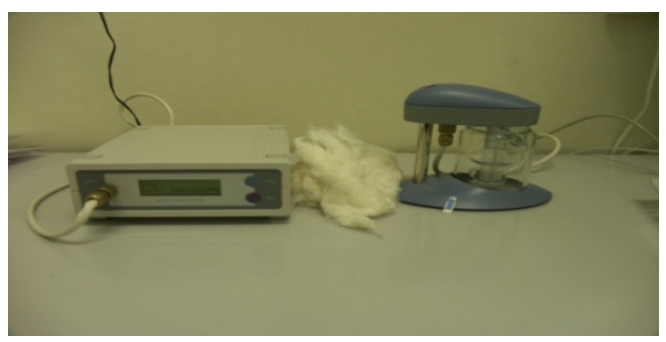

Figure 1. Biosensor toxicity analyzer with microflow unit.

\subsection{Reagents and Apparatus}

HPLC grades Hexane and dichloromethane solvents were used for the extraction procedure. Anhydrous and granular sodium sulfate was used as dehydrating agent throughout the extraction process. Glass wool was used as supporting material in soxhlet extraction after cleaned with acetone. A round flask $(250 \mathrm{ml})$ with soxhlet and condenser glassware was used to conduct the soxhlet extraction. MOPSO sodium salt was used for the preparation of buffer solution in BTA, where as Acetylthiocholine chloride (ATCCl) as enzyme substrate and Neostigmine methyl sulfate as enzyme inhibitor. AC1.W2.R1/ACCHE Sensors were used for the monitoring of $\mathrm{AChE}$ inhibition.

All the above experiments were done at the Technical University of Liberec, University of Pardubice and Bvt technologies, Czech Republic.

\subsection{Sample Preparation}

The determination of pesticides in samples at low concentrations is always a challenge. The main aim of any extraction process is the isolation of analytes of interest from the selected sample by using an appropriate extracting phase. The development of an appropriate sample preparation procedure involving extraction, enrichment, and cleanup steps becomes mandatory to obtain a final extract concentrated on target analytes. It is always necessary to carry out some pretreatments to get a homogeneous and representative subsample [4]. Even if the sample is apparently homogeneous, that is, an aqueous sample, it will be at least necessary to perform a filtration step to remove suspended particles, which could affect the final determination of target analytes [15].

\subsubsection{Cryogenic Homogenization}

Both samples of classical conventional cotton and organic cotton were arranged inside of a pre-chilled Teflon mill in the form of pallets which contains a concentric Teflon ring and Teflon puck in liquid nitrogen surrounding. Each sample was milled for approximately $10 \mathrm{~min}-$ utes with an interval of $2 \mathrm{~min}$ for grinding and $1 \mathrm{~min}$ for cooling. After the milling the resulting powder was sampled, cleaned and stored for analysis. Once the entire sample was homogenized and blended, the powder was 
sampled, cleaned and stored for analysis.

\subsubsection{Soxhlet Extraction (SOX)}

The Soxhlet Extraction method was used for the extraction from both of the samples. Two different solvents hexane and dichloromethane were used for each of the sample. A total of $1.0 \mathrm{gm}$ homogenized sample was transferred to the Soxhlet thimble in between two layers of dehydrated sodium sulfate over a glass wool layer. The thimble was placed in the extraction apparatus charged with $230 \mathrm{ml}$ of both the solvents, separately. Samples were extracted for overnight. The extract then concentrated by turbo evaporator and stored for further analysis.

Biosensor Toxicity Analyzer (BTA) equipped with AC1.W2.R1/ACCHE sensors was used for the monitoring of AChE inhibition. MOPSO sodium salt was used for the preparation of buffer solution in BTA, where as Acetylthiocholine chloride (ATCCl) as enzyme substrate and Neostigmine methyl sulfate as enzyme inhibitor.

\section{Results and Discussion}

All the above mentioned four extracts were injected in BTA for analysis. For the convenience we will describe the results with respect to the solvent used for the extraction process.

\subsection{Hexane}

Both the samples of classical cotton and organic cotton were tested on BTA. After putting the sensor in the slot, the buffer solution is added in the microflow unit and pump is started. After some stabilization the sample is added and then the inhibitor, Neostigmine methyl sulfate, is added in the solution to compare the inhibition of the sample with the standard inhibitor. The resultant graphs of the whole activity are shown in Figure 2 and Figure 3 for classical cotton and organic cotton, respectively. In these graphs the response of current (nano amperes) is on $\mathrm{Y}$ axis and the time (seconds) is on $\mathrm{X}$ axis.

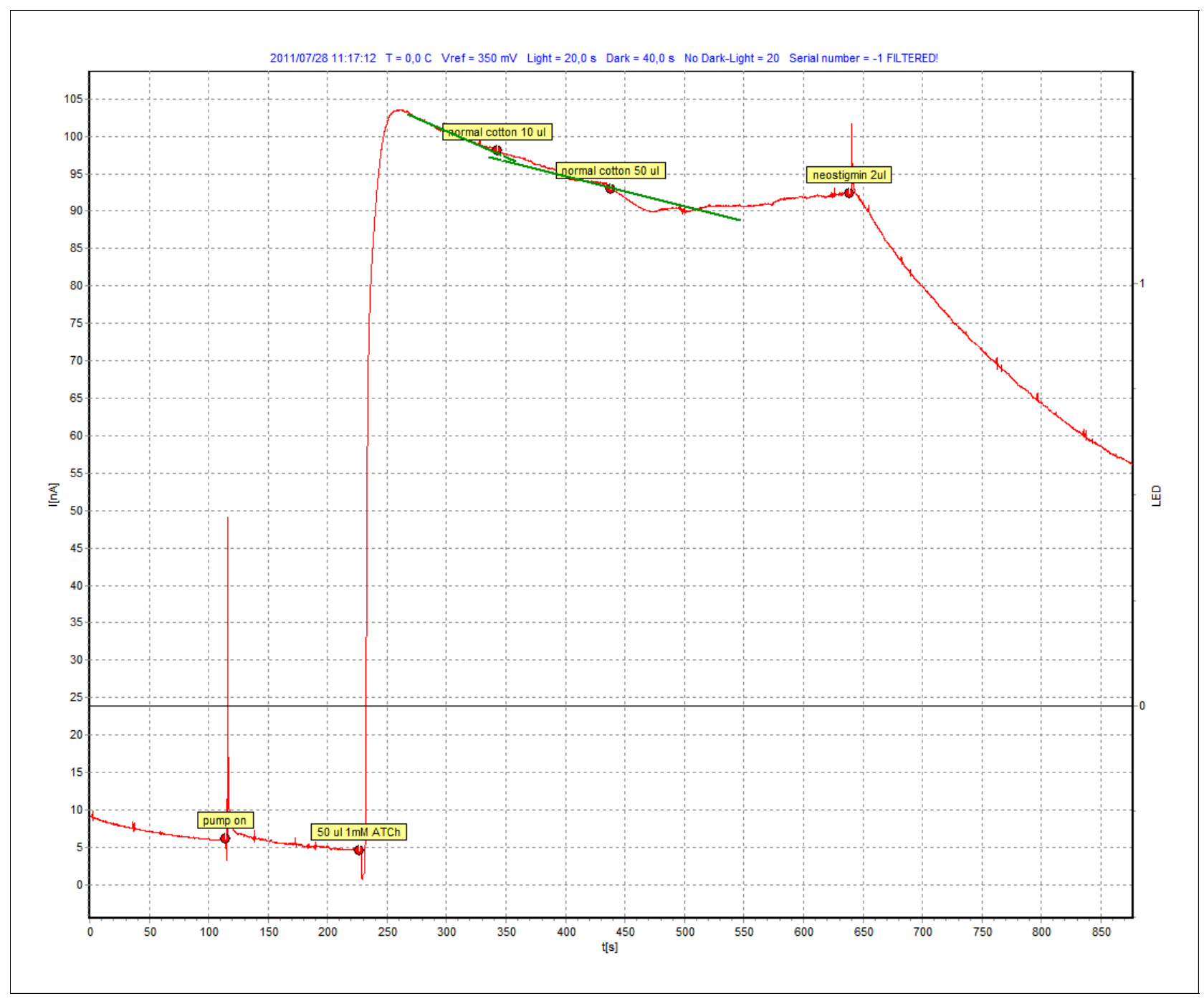

Figure 2. Classical cotton sample with hexane. 


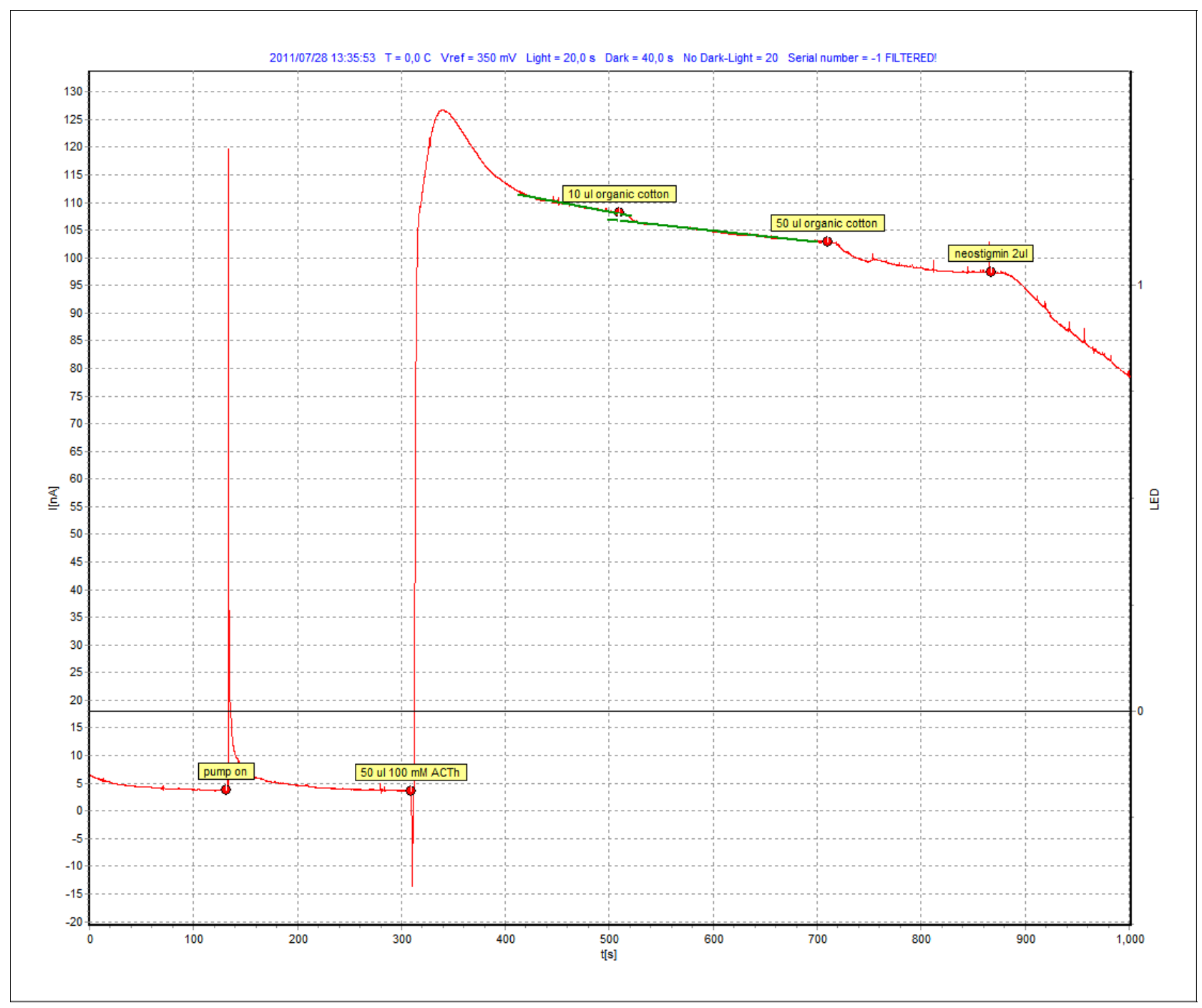

Figure 3. Organic cotton sample with hexane.

\subsection{Dichloromethane}

The same procedure as mentioned in the case of the solvent hexane is repeated for the second solvent i.e. dichloromethane and the resultant graphs are shown in Figures 4-5. It is quite visible in these graphs that there is a clear response on the addition of both the samples which can be compared with the slope of the standard inhibitor. We measure this response $(\Delta \mathrm{I})$ and also the relative inhibition $\left(\mathrm{R}_{\mathrm{i}}\right)$, which is calculated to quantify inhibiting effect of an inhibitor to the enzyme. Inhibiting effect is proportional to the slope of current time dependence after inhibitor or sample addition. The calculated values are shown in Table 1.

It can be observed that although both classical and organic cotton samples show the change in the intensity of the current but the organic cotton sample shows more response and more inhibition with each solvent. Although the use of synthetic pesticides and sprays are prohibited in the cultivation of organic cotton but the presence of these xenobiotic compounds indicate the improper storage, organic fields surrounded by the conventional cotton fields or may be some negligence in the organic cotton production line.

\section{Conclusions}

This study demonstrates a method based on AChE inhibition. Contrary to other sophisticated methods, this is an easier, faster and cheaper method. It is a method that offers to different investigators an easy way to detect the presence of organophosphorous and carbamate pesticides.

As there is worldwide interest in organic cotton as a potentially environmentally friendly way to produce cotton so this method can be helpful for analyzing the truth of the statement. Further research must be needed to verify the usefulness of the method presented here for the screening of pesticides on some more varieties of cotton of different regions. 


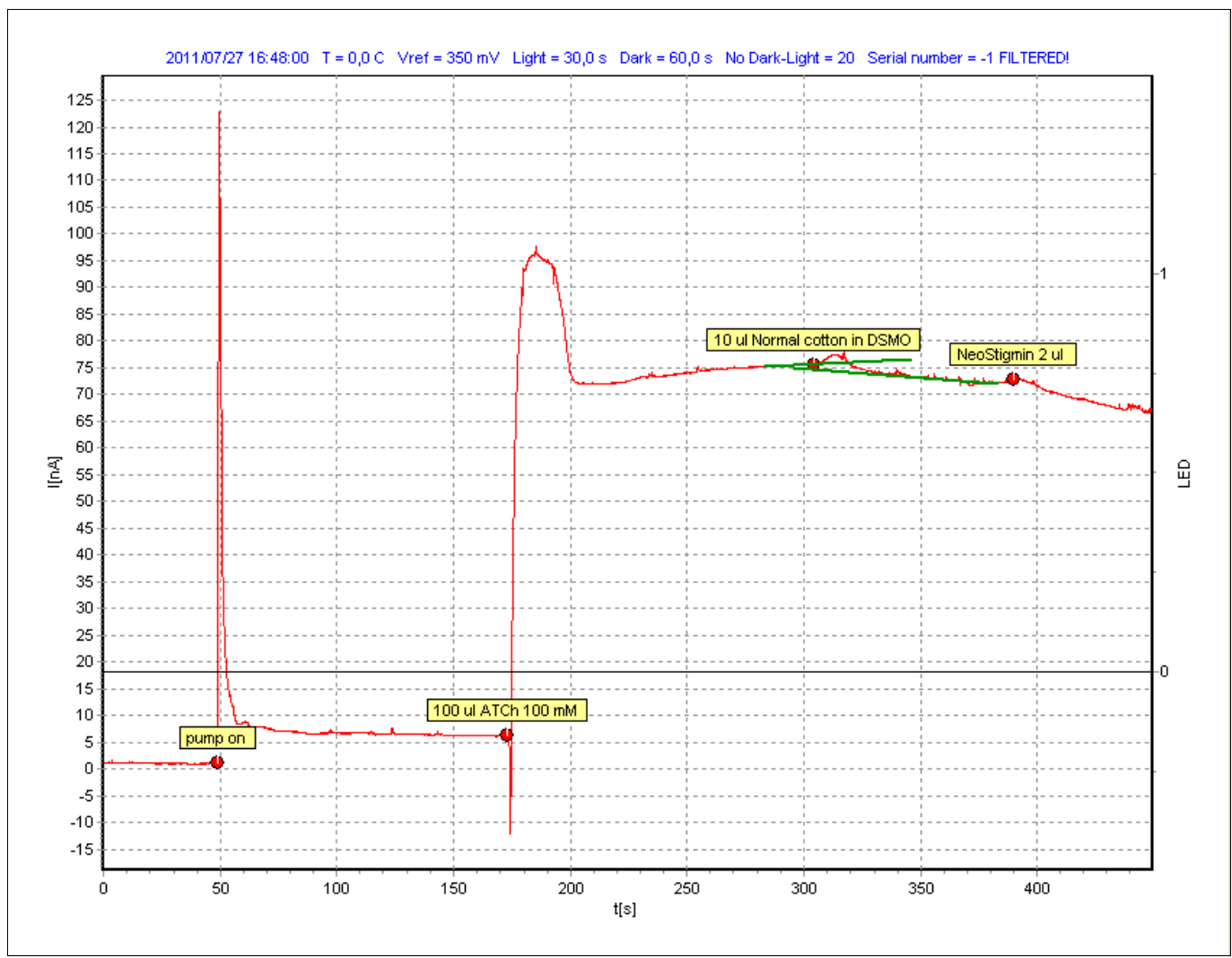

Figure 4. Classical cotton sample with DCM.

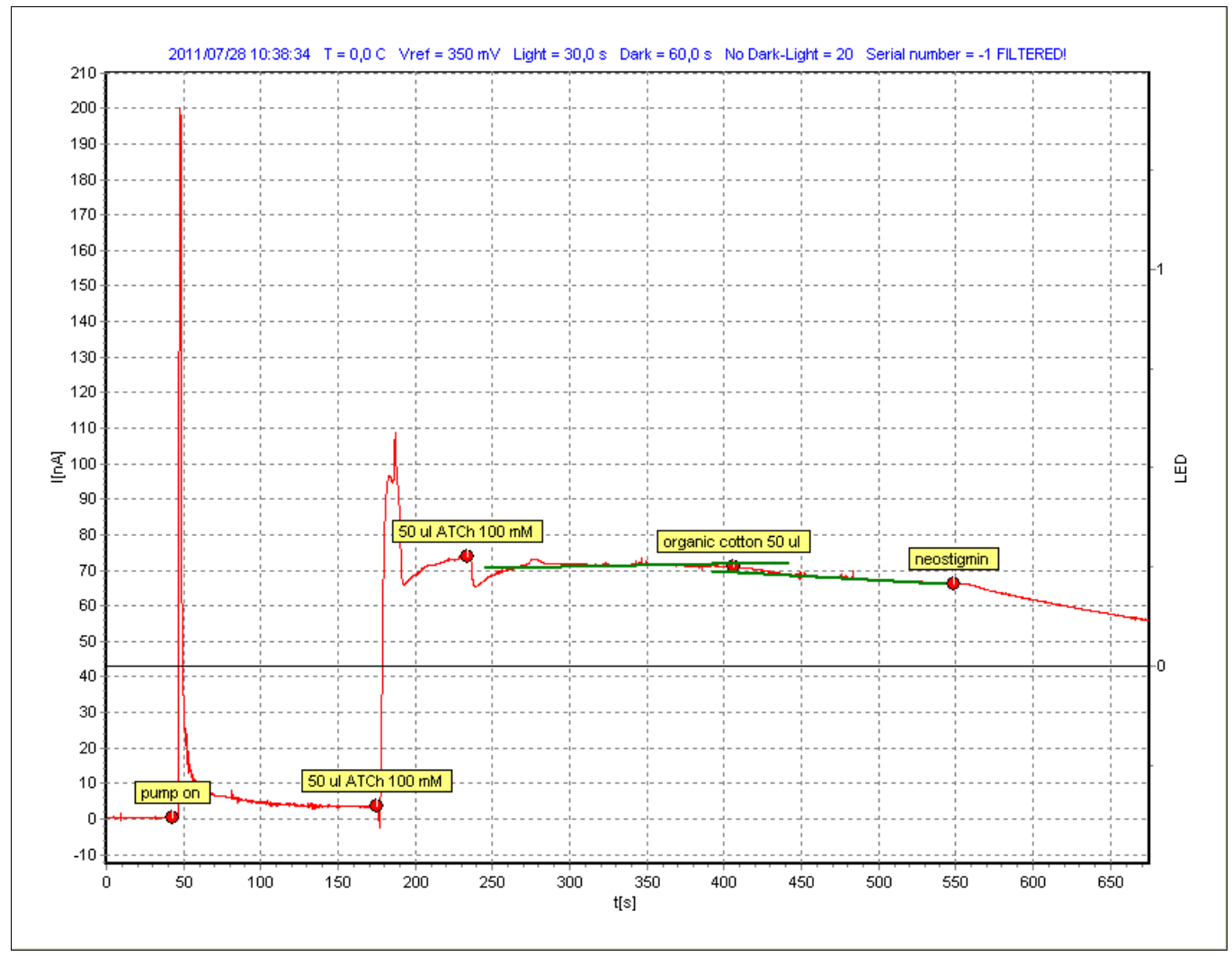

Figure 5. Organic cotton sample with DCM. 
Table 1. Response and Relative Inhibition for classical and organic cotton.

\begin{tabular}{ccccc}
\hline \multirow{2}{*}{ Solvents } & \multicolumn{2}{c}{$\begin{array}{c}\text { Classical conventional } \\
\text { cotton }\end{array}$} & \multicolumn{2}{c}{ Organic cotton } \\
\cline { 2 - 5 } & $\begin{array}{c}\text { Response } \\
{[\Delta \mathrm{I}(\mathrm{nA})]}\end{array}$ & $\begin{array}{c}\text { Relative } \\
\text { Inhibition }\left[\mathrm{R}_{\mathrm{i}}\right]\end{array}$ & $\begin{array}{c}\text { Response } \\
{[\Delta \mathrm{I}(\mathrm{nA})]}\end{array}$ & $\begin{array}{c}\text { Relative } \\
\text { Inhibition }\left[\mathrm{R}_{\mathrm{i}}\right]\end{array}$ \\
\hline Hexane & 0.6837 & -0.0002436 & 1.246 & -0.0000937 \\
Dichloromethane & 3.208 & -0.0004602 & 3.524 & -0.0003441 \\
\hline
\end{tabular}

\section{Acknowledgements}

This work was supported under Student Grant Scheme (SGS) by Technical University of Liberec, Czech Republic.

\section{REFERENCES}

[1] W. C. Robertson and B. A. Roberts, "Integrated Crop Management for Cotton Production in the 21st Century," In: M. R. Chaudhry and P. J. Wakelyn, Eds., COTTON: Technology for the 21st Century, First International Cotton Advisory Committee, Washington DC, 2010, pp. 63-98.

[2] S. Stilton, D. Myers, "Organic Cotton: A More Sustainable Approach," Organic Cotton, Intermediate Technology Publications Limited, London, 1999, pp. 1-7.

[3] L. Grose, "Sustainable Cotton Production," In: R. S. Blackburn, Ed., Sustainable Textiles: Life Cycle and Environmental Impact, Woodhead Publishing Limited, Cambridge, 2009, pp. 33-62.

[4] T. E. Martín-Esteban, "Sample Handling of Pesticides in Food and Environmental Samples," In: J. L. Tadeo, Ed., Analysis of Pesticides in Food and Environmental Samples, CRC Press, Boca Raton, 2008, pp. 35-58.

[5] J. W. S. Hearle, "Physical Structure and Properties of cotton," In: S. Gordon and Y.-L. Hsieh, Eds., Cotton: Science and Technology, Woodhead Publishing Limited, Cambridge, 2007, pp. 35-67.

[6] A. Lutz, "Residue Analysis of 500 High Priority Pesticides: Better by GC-Ms or LC-Ms/Ms?" Wiley InterScience, Vol. 25, No. 6, 2006, pp. 838-865. doi:10.1002/mas.20091

[7] A. El-Rahman El-Naggar, "Clinical Findings and Choli- nesterase Levels in Children of Organophosphates and Carbamates Poisoning," European Journal of Pediatrics, Vol. 168, No. 8, 2009, pp. 951-956. doi:10.1007/s00431-008-0866-Z

[8] H. Y. Hu, “A Novel Chemiluminescence Assay of Organophosphorous Pesticide Quinalphos Residue in Vegetable with Luminol Detection," Chemistry Central Journal, Vol. 4, 2010, p. 13. doi:10.1186/1752-153X-4-13

[9] A. Mulchandani, "Biosensors for Direct Determination of Organophosphate Pesticides," Biosensors \& Bioelectronics, Vol. 16, No. 4, 2001, pp. 225-230. doi:10.1016/S0956-5663(01)00126-9

[10] M. L. Hannam, "Characterisation of Esterases as Potential Biomarkers of Pesticide Exposure in the Lugworm Arenicola Marina (Annelida: Polychaeta)," Environmental Pollution, Vol. 152, No. 2, 2008, pp. 342-350. doi:10.1016/i.envpol.2007.06.022

[11] P. Mulchandani, "Biosensor for Direct Determination of Organophosphate Nerve Agents. 1. Potentiometric Enzyme Electrode," Biosensors \& Bioelectronics, Vol. 14, No. 1, 1999, pp. 77-85. doi:10.1016/S0956-5663(98)00096-7

[12] R. P. Deo, "Determination of Organophosphate Pesticides at a Carbon Nanotube/Organophosphorus Hydrolase Electrochemical Biosensor," Analytica Chimica Acta, Vol. 530, No. 2, 2005, pp. 185-189. doi:10.1016/j.aca.2004.09.072

[13] D. G. Buerk, "Biosensors: Theory and Applications," 1st Edition, Technomic Publishing Company, Lancaster, 1995.

[14] A. Adina, "Development of a Pesticides Biosensor Using Carbon-Based Electrode Systems," In: L. Simeonov and E. Chirila, Eds., Chemicals as Intentional and Accidental Global Environmental Threats, Springer, Dordrecht, 2006, pp. 337-343.

[15] J. L. Tadeo, "Pesticides: Classification and Properties," In: J. L. Tadeo, Ed., Analysis of Pesticides in Food and Environmental Samples, CRC Press, Boca Raton, 2008, pp. 1-34.

[16] A. Mulchandani, "Microbial Biosensors for Organophosphate Pesticides," Applied Biochemistry and Biotechnology, Vol. 165, No. 2, 2011, pp. 687-699.

doi:10.1007/s12010-011-9288-x 